\title{
Large Polarization Squeezing in Non-Degenerate Parametric Amplification of Coherent Radiation
}

\author{
Namrata Shukla ${ }^{1,2, *}$ and Ranjana Prakash ${ }^{2}$ \\ ${ }^{1}$ Now at, Quantum Information and Computation Group, \\ Harish-Chandra Research Institute, Chhatnag Road, Jhunsi, Allahabad 211 019, India \\ ${ }^{2}$ Department of Physics, \\ University of Allahabad, Allahabad, Allahabad-211001, UP, India
}

(Dated: March 26, 2018)

\begin{abstract}
Polarization squeezing is shown to occur in non-degenerate parametric amplification of coherent light and the degree of squeezing at interaction time $T$ can be as large as $1-e^{-2 T}$. This gives $86.4 \%$ polarization squeezing for $T=1$ and $98.2 \%$ for $T=2$. One simple case when this occurs is on taking initially plane polarized light having equal amplitudes in the two modes that finally has equal intensities of two circular polarizations. This suggest the experimental settings of parameters to achieve this extent of polarization squeezing in coherent light.

PACS numbers: Valid PACS appear here
\end{abstract}

\section{INTRODUCTION}

Polarization of light is a concept receiving attention since very long and the mathematically convenient way of describing partial polarization was given classically with the help of Stokes parameters [1, 2]. For a monochromatic unidirectional light traveling along z-direction, Stokes parameters denoted by $S_{0}$ and $\boldsymbol{S}=S_{1}, S_{2}, S_{3}$ are defined as

$$
S_{0,1}=\left\langle\mathcal{E}_{x}^{*} \mathcal{E}_{x}\right\rangle \pm\left\langle\mathcal{E}_{y}^{*} \mathcal{E}_{y}\right\rangle, S_{2}+i S_{3}=2\left\langle\mathcal{E}_{x}^{*} \mathcal{E}_{y}\right\rangle,
$$

where $\mathcal{E}_{x, y}$ are the components of analytic signal [3] for the electric field. For perfectly polarized light

$$
S_{0}^{2}=|\boldsymbol{S}|^{2}=S_{1}^{2}+S_{2}^{2}+S_{3}^{2} .
$$

The point having coordinates $\left(S_{1}, S_{2}, S_{3}\right)$ lies on a sphere of radius $S_{0}$ called Poincare sphere. For unpolarized light, $|\boldsymbol{S}|=$ 0 and for partially polarized light, $S_{0}>|\boldsymbol{S}|$.

Relevant continuous variables for the treatment of quantum nature of polarization of the system are called quantum Stokes operators [4]. Quantum Stokes operators and the associated Poincare sphere describes the quantum nature of polarization of light. In direct analogy with the classical Stokes parameters, these Stokes operators $\hat{S}_{0}$ and $\hat{\boldsymbol{S}}=\hat{S}_{1,2,3}$ are defined in terms of creation and annihilation operators as

$$
\hat{S}_{0,1}=\hat{a}_{x}^{\dagger} \hat{a}_{x} \pm \hat{a}_{y}^{\dagger} \hat{a}_{y}, \hat{S}_{2}+i \hat{S}_{3}=2 \hat{a}_{x}^{\dagger} \hat{a}_{y} .
$$

These Stokes operators involve the coherence functions [5] of order $(1,1)$ and shown to be insufficient to describe polarization completely as $\boldsymbol{S}=0$ does not represent only the unpolarized light [6]. These operators however, remain important because of the non-classicalities like polarization squeezing and polarization entanglement associated with polarization.

Commutation relations $\left[\hat{a}_{j}, \hat{a}_{k}^{\dagger}\right]=\delta_{j k}$ of annihilation and creation operators lead to the commutation relations

$$
\left[\hat{S}_{0}, \hat{S}_{j}\right]=0,\left[\hat{S}_{j}, \hat{S}_{k}\right]=2 i \sum_{l} \epsilon_{j k l} \hat{S}_{l},
$$

$\epsilon_{j k l}$ being Levi-Civita symbol for $(j, k, l=1,2$, or 3$)$. The quantum fluctuations of these Stokes operators are bounded below by following uncertainty relations

$$
V_{j} V_{k} \geqslant\left\langle\hat{S}_{l}\right\rangle^{2}, V_{j} \equiv\left\langle\hat{S}_{j}^{2}\right\rangle-\left\langle\hat{S}_{j}\right\rangle^{2}
$$

Squeezed radiation states in quantum optics are identified by the property that their quantum fluctuations are reduced below the standard quantum limit in one of the quadrature components. Polarization squeezing [7] is defined using above mentioned commutation relations and uncertainty products for Stokes operators in a similar pattern. Polarization squeezing is important in quantum information theory and it is desirable to devise methods for generation of states with appreciable amount of polarization squeezing.

In a study by Heersink et al. [8], polarization squeezing has been investigated by taking both the polarization modes of coherent light to be amplitude squeezed. $\hat{S}_{1}$ is found to be polarization squeezed by $-3.4 \mathrm{~dB}$ as per the definition of polarization squeezing given in the same paper and it was also experimentally investigated.

In this paper, we investigate the polarization squeezing using the most general criterion, in the coherent light subjected to non-degenerate parametric amplification which is never reported before as a closed form result. Parametric amplification is used to generate high polarization squeezing which is more than earlier reported cases for considerably small times of interaction. The experimental feasibility of the technique with suitable fixing of parameters is discussed in section IV.

Coinciding with the above reported paper by Heersink et.al., this study reveals the polarization squeezing only along $\hat{S}_{1}$ component of Stokes vector. We quantify the polarization squeezing by defining Squeezing factor and Degree of squeezing. Minimum value of squeezing factor exhibits the maximum squeezing as a function of interaction time. 


\section{CRITERION FOR POLARIZATION SQUEEZING}

Polarization squeezing was first defined by Chirkin et al. [9] in terms of variances of stokes operators for a given state as

$$
V_{j}<V_{j}(\operatorname{coh})=\hat{S}_{0}
$$

,i.e., $\hat{S}_{j}$ is squeezed if the variance of Stokes parameter is less than that for an equally intense coherent state.

Heersink et al. defined polarization squeezing in their earlier mentioned paper using the uncertainty relations (5) in the form

$$
V_{j}<\left|\left\langle\hat{S}_{l}\right\rangle\right|<V_{k} \text { for } j \neq k \neq l,
$$

for squeezing of $\hat{S}_{j}$.

Luis and Korolkova [10] then considered various criteria for polarization squeezing and their stringency was compared. They give the following criterion for polarization squeezing of a component of $\hat{\boldsymbol{S}}$ along a unit vector $\boldsymbol{n}$ as

$$
V_{n}<\left|\left\langle\hat{S}_{n_{\perp}}\right\rangle\right|,
$$

where $\hat{S}_{\boldsymbol{n}_{\perp}}$ is component of $\hat{\boldsymbol{S}}$ along unit vector $\boldsymbol{n}_{\perp}$ which is perpendicular to $\boldsymbol{n}$. For suitable orthogonal components $\hat{S}_{\boldsymbol{n}}$ and $\hat{S}_{\boldsymbol{n}_{\perp}}$, they have discussed the order of stringency of the various criteria

$$
V_{\boldsymbol{n}}<\left\langle S_{\boldsymbol{n}_{\perp}}\right\rangle^{2} /\left\langle\hat{S}_{0}\right\rangle<\left|\left\langle S_{\boldsymbol{n}_{\perp}}\right\rangle\right|<\left\langle\hat{S}_{0}\right\rangle .
$$

The authors [11] finally write the criterion for polarization squeezing in the form

$$
\begin{aligned}
V_{\boldsymbol{n}} \equiv\left\langle\Delta S_{\boldsymbol{n}}^{2}\right\rangle\left\langle\left|\left\langle S_{\boldsymbol{n}_{\perp}}\right\rangle\right|_{\max }\right. & \\
= & \sqrt{|\langle\hat{\boldsymbol{S}}\rangle|^{2}-\left\langle S_{\boldsymbol{n}}\right\rangle^{2}}
\end{aligned}
$$

arguing that for a given component $\hat{S}_{n}$ there are infinite directions $\boldsymbol{n}_{\perp}$ and therefore it is required to consider the maximum possible value of $\left|\left\langle\hat{S}_{n_{\perp}}\right\rangle\right|$. Eqs. (6) - (8) and (11) describes non-classicality but the uncertainty relations are considered only in (7), (8) and (11).

We shall use the criterion (11) for polarization squeezing which is most general and based on the actual uncertainty relations. We may define squeezing factor $\mathcal{S}_{\boldsymbol{n}}$ and degree of squeezing $\mathcal{D}_{n}$ by writing

$$
\mathcal{S}_{n}=\frac{V_{n}}{\sqrt{|\langle\hat{\boldsymbol{S}}\rangle|^{2}-\left\langle S_{n}\right\rangle^{2}}}, \mathcal{D}_{n}=1-\mathcal{S}_{\boldsymbol{n}} .
$$

Non-classicalities appear when $1>\mathcal{S}_{\boldsymbol{n}}>0$ and the degree of squeezing $\mathcal{D}_{\boldsymbol{n}}$ lies between 0 and 1 .

\section{HAMILTONIAN AND POLARIZATION SQUEEZING}

The hamiltonian [12] for non-degenerate parametric amplification of light traveling along z-direction is given by

$$
H=k\left(\hat{a}_{x}^{\dagger} \hat{a}_{y}^{\dagger}+\hat{a}_{x} \hat{a}_{y}\right),
$$

$k$ being coupling constant and $\hat{a}_{x, y}$ are annihilation operators for the two linear polarizations $x$ and $y$. Solution to this hamiltonian [12] is given by

$$
\begin{aligned}
& \hat{a}_{x}(t)=(\cosh k t) \hat{a}_{x}-i(\sinh k t) \hat{a}_{y}^{\dagger}, \\
& \hat{a}_{y}(t)=(\cosh k t) \hat{a}_{y}-i(\sinh k t) \hat{a}_{x}^{\dagger} .
\end{aligned}
$$

If we consider the incident light in the coherent state $|\alpha, \beta\rangle$, where $\alpha=A \cos \theta e^{i \phi_{x}}, \beta=A \sin \theta e^{i \phi_{y}}$, straight forward calculations give the expectation values and variances of Stokes parameters at the interaction time $T \equiv k t$ as

$$
\begin{aligned}
\left\langle\hat{S}_{1}(T)\right\rangle= & A^{2} \cos 2 \theta, \\
\left\langle\hat{S}_{2}(T)\right\rangle= & A^{2}\left[\cosh 2 k t \sin ^{2} \theta \cos \left(\phi_{x}-\phi_{y}\right)\right. \\
& \left.-\sinh 2 k t\left(\cos ^{2} \theta \sin 2 \phi_{x}+\sin ^{2} \theta \sin 2 \phi_{y}\right)\right], \\
\left\langle\hat{S}_{3}(T)\right\rangle= & A^{2}\left[-\cosh 2 k t \sin ^{2} \theta \sin \left(\phi_{x}-\phi_{y}\right)\right. \\
& \left.-\sinh 2 k t\left(\cos ^{2} \theta \cos 2 \phi_{x}-\sin ^{2} \theta \cos 2 \phi_{y}\right)\right],
\end{aligned}
$$

and

$$
\begin{aligned}
V_{1}(T)= & A^{2}, \\
V_{2}(T)= & V_{3}(T) \\
= & A^{2} \cosh ^{2} 2 k t+\sinh ^{2} 2 k t\left(A^{2}+1\right) \\
& -A^{2} \sinh 4 k t\left(c^{2}+s^{2}\right) \sin 2 \theta \sin \left(\phi_{x}+\phi_{y}\right),
\end{aligned}
$$

where $c=\cosh k t, s=\sinh k t$.

As per the criterion given by Eq. 111, a glance at expressions for $V_{1}$ and $\left\langle\hat{S}_{2,3}\right\rangle$ gives an idea that component $\hat{S}_{1}$ can be squeezed. The squeezing factor in this case takes the form

$$
\mathcal{S}_{1}=\frac{A^{2}}{A^{2} \sqrt{R}}
$$

where

$$
\begin{aligned}
R= & \frac{\sqrt{\left\langle\hat{S}_{2}\right\rangle^{2}+\left\langle\hat{S}_{3}\right\rangle^{2}}}{A^{2}} \\
= & \cosh ^{2} 2 k t \sin ^{2} 2 \theta+\sinh ^{2} 2 k t\left(\sin ^{4} \theta+\cos ^{4} \theta\right) \\
& -2 \sinh ^{2} 2 k t \sin ^{2} \theta \cos ^{2} \theta \cos 2\left(\phi_{x}+\phi_{y}\right) \\
& -\sinh 4 k t \sin 2 \theta \sin \left(\phi_{x}+\phi_{y}\right) .
\end{aligned}
$$

In order to achieve maximum squeezing we need to minimize the squeezing factor $\mathcal{S}_{1}$ and thus maximize $R$. A slight manipulation lets $R$ be written as

$$
R=\left[\cosh 2 T-\sinh 2 T \sin 2 \theta \sin \left(\phi_{x}+\phi_{y}\right)\right]^{2}-\cos ^{2} 2 \theta .
$$


This gives maximum value of $R$ and hence minimum value of squeezing factor $\mathcal{S}_{1}$ denoted by $\mathcal{S}_{1 \text { min }}$, for $\theta=\pi / 4$ and $\left(\phi_{x}+\phi_{y}\right)=3 \pi / 2$ in their admissible range of values. It leads to,

$$
\mathcal{S}_{1 \min }=e^{-2 T} \text {. }
$$

$$
\phi_{x}+\phi_{y}
$$

$2 \pi$
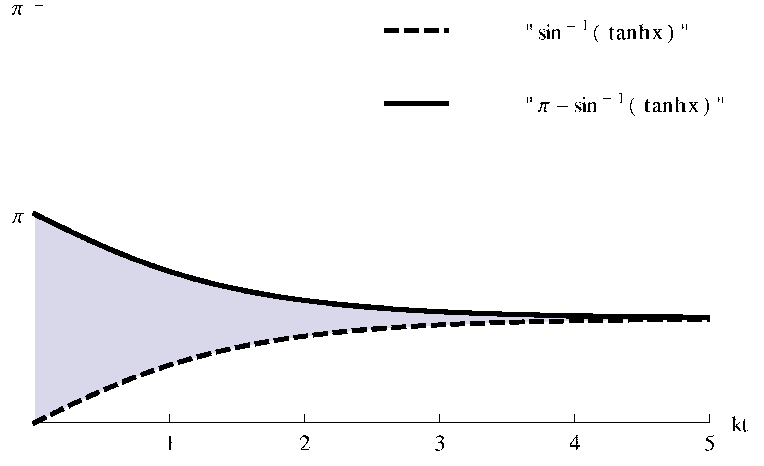

FIG. 1. Region of squeezing for $\phi_{x}+\phi_{y}$ with $k t$. Squeezing occurs outside the region between the curves

\section{RESULT AND DISCUSSION}

The maximum degree of polarization squeezing is $\mathcal{D}_{1 \max }=1-e^{-2 T}$ for $\theta=\pi / 4$ and $\left(\phi_{x}+\phi_{y}\right)=3 \pi / 2$. This gives a very large amount of polarization squeezing at moderate interaction times $T$. For $T=1$, we get $\mathcal{D}_{1}=0.864$, i.e., $86.4 \%$ polarization squeezing and for $T=2, \mathcal{D}_{1}=0.982$, i.e., $98.2 \%$ polarization squeezing is obtained.

To adjust $\theta=\pi / 4$ in experiments is easy as this requires only the equal intensities of $x$ and $y$ components, i.e., $|\alpha|=$ $|\beta|$. On this condition being followed alone, polarization squeezing is seen for $\sqrt{R}=\left[\cosh 2 k t-\sinh 2 k t \sin \left(\phi_{x}+\right.\right.$ $\left.\left.\phi_{y}\right)\right]>1$ or $\sinh \left(\phi_{x}+\phi_{y}\right)<\tanh k t$.

We plot $\left(\phi_{x}+\phi_{y}\right)$ against $k t$ [Figure 1] and it shows that polarization squeezing occurs for all values $\left(\phi_{x}+\phi_{y}\right)$ except those between $\phi_{1}=\sin ^{-1}(\tanh k t)$ and $\phi_{2}=\pi-$ $\sin ^{-1}(\tanh k t)$, which is very narrow for appreciable values of interaction time. For $\theta=\pi / 4,\left\langle\hat{S}_{2}(T)\right\rangle$ and $\left\langle\hat{S}_{3}(T)\right\rangle$ take the form,

$$
\begin{aligned}
& \left\langle\hat{S}_{2}(T)\right\rangle=A^{2} \sinh 2 k t \cos \left(\phi_{x}-\phi_{y}\right), \\
& \left\langle\hat{S}_{3}(T)\right\rangle=-A^{2} \sin \left(\phi_{x}-\phi_{y}\right) e^{-2 k t} .
\end{aligned}
$$

If we choose $\phi_{x}=\phi_{y}, \hat{S}_{2}(T)$ will have maximum value and $\hat{S}_{3}(T)$ will vanish. An experimentalist may therefore take plane polarized light along the direction dividing $x$ and $y$ directions ensuring $\phi_{x}=\phi_{y}$ and thus $\theta=\pi / 4$ and then vary the input phase $\phi_{x}=\phi_{y}$ so as to make

1. $\hat{S}_{2}(t)=I_{+}-I_{-}$, where $I_{+}$and $I_{-}$are intensities of plane polarized components along directions midway between $x$ and $y$ directions.

2. $\hat{S}_{3}(t)=I_{R}-I_{L}=0$, where $I_{R}$ and $I_{L}$ are intensities of right handed and left handed circularly polarized components.

This will make $\left(\phi_{x}+\phi_{y}\right)=3 \pi / 2$ and the output will show maximum polarization squeezing.

\section{ACKNOWLEDGEMENT}

We would like to thank Hari Prakash and Naresh Chandra for their interest and critical comments.

* namratashukla@hri.res.in

\section{REFERENCES}

[1] G. G. Stokes, Trans.Cambridge Phylos. Soc.9, 399 (1852).

[2] M. Born and E. Wolf, Principles of Optics (Cambridge Univ. Press, Cambridge, 1999).

[3] See, e.g., ref.[2].

[4] L. Mandel and E. Wolf, Optical Coherence and Quantum Optics (Cambridge Univ. Press, Cambridge, 1995).

[5] See, e.g., ref.[4].

[6] H. Prakash and N. Chandra, Phys. Rev. A 4, 796 (1971); H. Prakash and N. Chandra, Phys. Rev. A 9, 1021 (1974).

[7] A. S. Chirkin and V. V. Volokhovsky, Journal of Russian Laser Research 16, 6 (1995); N. Korolkova and R. Loudon, Phys. Rev. A 71, 032343 (2005).

[8] J. Heersink, T. Lorenz, O. Glock1, N. Korolkova,and G. Leuchs, Phys. Rev. A 68, 013815 (2003).

[9] A. S. Chirkin, A. A. Orlov, and D. Y. Parashchuk, Quantum Electron 23, 870 (1993).

[10] A. Luis and N. Korolkova, Phys. Rev. A 74, 043817 (2006).

[11] R. Prakash and N. Shukla, Optics Communications 284, 3568 (2011).

[12] M. O. Schully and M. S. Zubairy, Quantum Optics (Cambridge Univ. Press, Cambridge, 1997). 